\title{
Develop Sonic Infrared Imaging NDE for Quantitative Assessment on Damage in Aircraft Composite Structures
}

\author{
by X. Han*, S. Zhao**, D. Zhang*, A. Lubowicki*, L. Favro***, R. Thomas**, and G. Newaz** \\ Department of Electrical and Computer Engineering, Wayne State University, Detroit, MI 48202, \\ USA, xhan@eng.wayne.edu \\ ** Department of Mechanical Engineering, Wayne State University, Detroit, MI 48202, USA \\ ${ }^{* * *}$ Department of Physics and Astronomy, Wayne State University, Detroit, MI 48202, USA
}

\begin{abstract}
Sonic IR imaging has been shown great advantages as a NDE technology to detect defects such as cracks in metal/metal alloy structures. However, composites cannot simply be regarded as "black aluminum". The acoustic behavior of composites is quite different from that of metals. This study is to aim at the understanding of the effect of the structures and the relative positions of the sound source and the defect. In this paper, we present our work on developing Sonic IR imaging NDE for aircraft composite structures.
\end{abstract}

\section{Introduction}

Composites have long been used in peripheral aircraft structures such as flaps and spoilers, and in some instances in the empennage and low stress fuselage areas. Sonic IR imaging combines ultrasound excitation of a short pulse (typically a fraction of a second) and wide-area infrared imaging. It has been shown its advantages as a fast, wide-area NDE technology to detect defects such as cracks, delamination, and disbonds in materials and structures [18]. Even though Sonic IR imaging is the youngest member in the NDE tools, research and development on this technology have been carried out by several groups of researchers in the recent years [9-19]. It is learned that the acoustic behavior of composites is quite different from that of metals. In addition, concerns of types of damage in those structures are quite different as well. In composites, disbonds and delaminations are some major concerns. Those types of damage are very often subsurface defects located at certain depths [20-25]. In the previous work on Sonic IR Imaging with composite materials, some of the authors had worked on some small-scale composite samples [7-11]. Ultrasound waves attenuate much more in composite materials such as carbon fiber reinforced laminated structures than in metal/metal alloy structures [23]. Thus, sound energy in composites does not propagate as far as it does in metal/metal alloy structures. Meanwhile, heating patterns in the background and interference patterns can occur as well [23]. These results can very often cause problems in defect identification in composites. However, by applying chaotic, instead of monochromatic, sound, background heat patterns can be greatly reduced, which can be greatly increase the detectability of defects in composites [24]. In spite of the attenuation in the course of ultrasound energy propagation, Sonic IR Imaging still has the capability of fast wide-area inspection in composite materials and structures. In this paper, we are going to present our development of Sonic Infrared Imaging for identifying defects in aircraft composite structures.

\section{Experimental arrangement and sample description}

In Sonic IR Imaging, the major pieces of equipment are an ultrasound excitation system, which contains an ultrasound transducer, power/control components, a commercial infrared imager, electronic synchronization control unit, and a computer with image acquisition and image/data processing unit. Ultrasound excitation sources provide a low frequency ultrasound pulse, typically a fraction of a second, to the target to cause relative motion of the faces of defects and thus frictional heating is produced in the defects. The temperature gradient in the sample results in the spreading of heat from the defects to their vicinity governed by heat diffusion equation. As a result, the surface temperature over the sample may rise which can be mapped by the infrared imager. In our experimental set-up, the ultrasound excitation systems we have used are Branson $20 \mathrm{kHz}$ ultrasound welder, and the IR imager is a FLIR commercial mid-IR camera. The number of the camera pixels and the size of the imaged area determine the spatial resolution of the details. The sample we used for this study is an A330 vertical stabilizer simulation panel. Figure 1 is a $3 D$ drawing that shows the backside structure of the panel with four stringers (ribs). The panel is $162 \mathrm{~cm} \times 58 \mathrm{~cm}$. The thickness of its skin is 0.41 $\mathrm{cm}$ and that over the stringers is $0.53 \mathrm{~cm}$. The adjacent stringers are about $16.5 \mathrm{~cm}$ apart. In addition, the panel also has four stiffeners as shown, and two of them contain disbonds. As seen in the drawing, they are located in two "channels" formed by two stringers. Each pair of the stiffeners is $70 \mathrm{~cm}$ apart. 


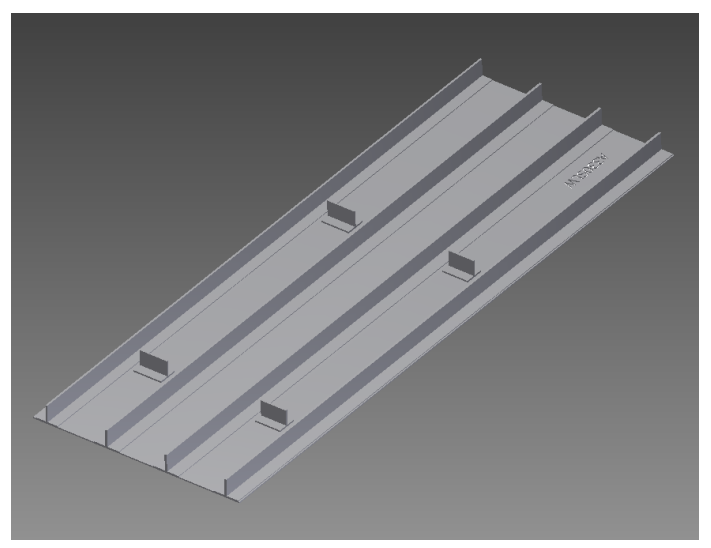

Fig. 1. $3 D$ Drawing showing the backside of the $A 330$ panel which has four stringers (ribs).

\section{Effect of the structures and the relative positions of the sound source and the defect}

The sample we used in this study is a simulation panel of A330 vertical stabilizer. We experienced the appearance differences in the resulting images of the two disbonded stiffeners when ultrasound energy is injected at different locations in the panel. When the ultrasound source was placed in the channels between two stringers where the two disbonded stiffeners are not located, we do not have the signal level from the IR camera to be able to declare the disbonds detected confidently. This is because the sound energy is basically confined in the channel between the stringers and the channel acts like a waveguide. When the ultrasound energy is injected in the channel where the defects are located, the disbands can be detected with certain level of IR signals. Therefore, the detectability of defects in composite materials can be greatly affected by their actual structures. However, even within the same channel, we can show that the relative positions of the ultrasound source and a defect can be critical for the detectability of the defect. Three particular locations were chosen for the transducer at the front side of the panel indicated with a red dot in the drawing in Figures $2 a, 2 b$ and $2 c$. IR images were taken from the front side of the panel for each corresponding experiments. In Figure $2 \mathrm{a}$, the transducer was put at the top end. The transducer is about $25 \mathrm{~cm}$ away from the closer stiffener. One can see that the IR image shows clearly the disbonded stiffeners located in the backside of the A330 panel circled in yellow. In Figure $2 b$, the transducer was put at the lower end of the panel, the IR image also shows the disbanded stiffeners, but the defects did not show as well as the results from the setting of Figure $2 \mathrm{a}$. In Figure $2 \mathrm{c}$, the transducer was put in between the two stiffeners. The IR image also shows the disbonded stiffeners, and showed the similar quality image to the setting of Figure $2 a$. 


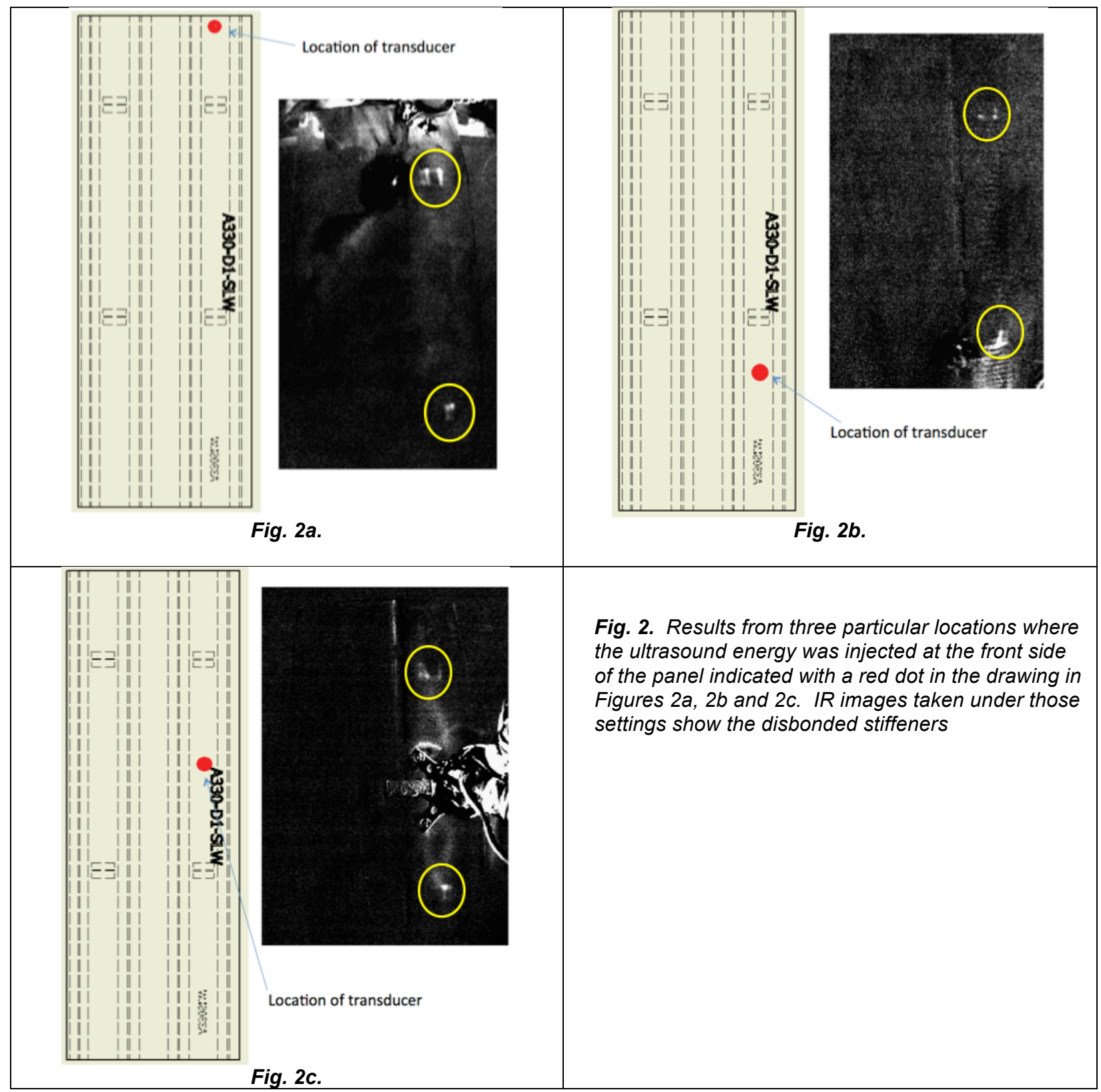

Based on the results shown above, further study was carried out. We set the sound pulse to $0.8 \mathrm{~s}$ in the experiments. This pulse length is relatively long compare to a typical pulse used in metal structures. This is due to the attenuation of the sound propagation in composites. In Figure 3, an IR image presents the disbonded stiffeners marked as "Spot 0" and "Spot 1". In the same figure, also presented is a drawing showing the positions of the disbonded stiffeners at "Spot 0" and "Spot 1" with green spots. Meanwhile, three red spots labeled as position 1, 2, and 3 are the positions at which a $20 \mathrm{kHz}$ ultrasound transducer was put to induce the ultrasound pulse for different experiments respectively. This study is to aim at the understanding of the effect of the structures and the relative positions of the sound source and the defect with quantitative measurement of the IR signal levels at the two spots with different sound source locations. Position 1 is $20 \mathrm{~cm}$ away from "spot 0 " to its right, and $90 \mathrm{~cm}$ away from "Spot 1" to its right; Position 2 is $50 \mathrm{~cm}$ away from "spot 0" to its left, and $20 \mathrm{~cm}$ away from "Spot 1" to its right; Position 3 is $119 \mathrm{~cm}$ from "spot 0 " to its left, and $50 \mathrm{~cm}$ away from "Spot 1" to its left. Please note that "Spot 0" and "spot 1" are $70 \mathrm{~cm}$ apart. The relative locations between the ultrasound source positions and the stiffeners are listed in Table 1. At each position of the sound source, the IR contrast signal levels for "Spot 0" and "Spot 1" resulted from a 3x3 pixel average are plotted for comparison, which represent the difference of IR radiation between the region of interest and the background region as a reference. All the IR signals were obtained at thirty frames per second for total of 10 seconds since the low diffusivity 
of the composite and the location depth of the stiffeners in the panel. Figure $4 \mathrm{a}$ and Figure $5 \mathrm{a}$ show the contrast IR signals on "Spot 0" and "Spot 1" respectively. The data clearly show how the signal levels are affected by the relative positions of the defect and the ultrasound excitation source. Among the first group of data for "Spot 0", the IR signal is at its highest when the transducer is put at position 1. Among the second group of data for "Spot 1", the IR signal is at its highest when the transducer is put at position 2. The IR signals from all the three positions at the two stiffeners fluctuate. Curve fitting of the experimental data was done to offset this fluctuation from noise. The $5^{\text {th }}$ degree polynomial was applied according to the expression (1) to fit all the 300 data for each of the six curves in a least-squares sense. The curve fitted plots are shown in Figure $4 \mathrm{~b}$ and Figure $5 \mathrm{~b}$ that represent the IR signal changes for all the three positions at the two stiffeners. It is clearly shown that the signal levels ranges from high to low according to the positions in the order of 1, 2, 3 at "Spot 0", and in the order of 2, 3, 1 at "Spot 1". These results are summarized in Table 1 as the "maximum IR contrast" as well. These results are quite different from those experienced in metal/metal alloy materials with Sonic IR Imaging. From all of the plots we see that they peaked around and after 4.7 seconds, fairly long after the end of the sound pulse at 0.8 second. The stiffeners are bonded to the backside of the panel and thus the disbonds occur under the panel skin thickness, which resulting in a much later peak time in these cases.

$$
y=a x^{5}+b x^{4}+c x^{3}+d x^{2}+e x+f
$$

口

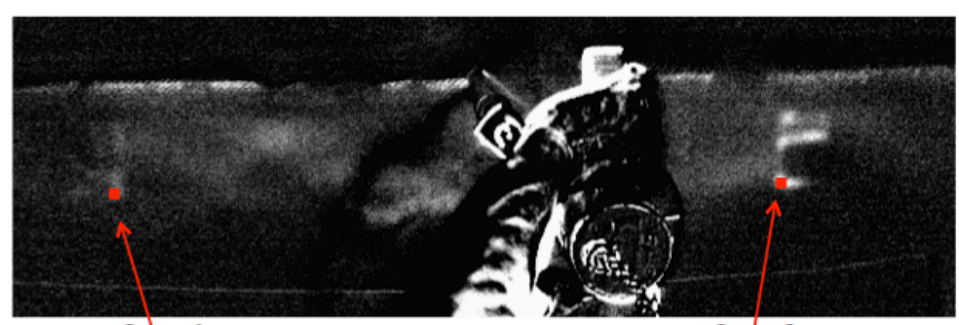

Spot 1

Spot 0

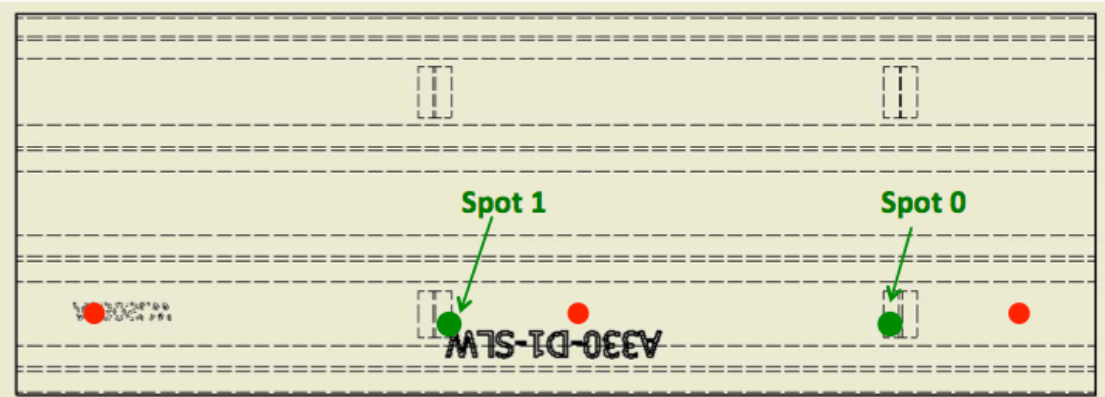

Position 3

Position 2

Position 1

Fig. 3. Drawing indicates the three locations where a $20 \mathrm{kHz}$ ultrasound transducer was put relative to the disbonded stiffeners labeled as "Spot 0" and "Spot 1".

Table 1. Relative locations between the ultrasound source positions and the stiffeners

\begin{tabular}{|c|l|c|c|c|}
\hline \multicolumn{2}{|c|}{} & Position 1 & Position 2 & Position 3 \\
\hline \multirow{3}{*}{ Spot 0 } & Relative Distance & $20 \mathrm{~cm}$ & $50 \mathrm{~cm}$ (Left) & $120 \mathrm{~cm}$ (Left) \\
\cline { 2 - 5 } & Max IR contrast & 49 & 22 & 3 \\
\hline \multirow{3}{*}{ Spot 1} & Relative Distance & $90 \mathrm{~cm}$ & $20 \mathrm{~cm}$ & $50 \mathrm{~cm}$ (Left) \\
\cline { 2 - 5 } & Max IR contrast & 7 & 38 & 16 \\
\hline
\end{tabular}




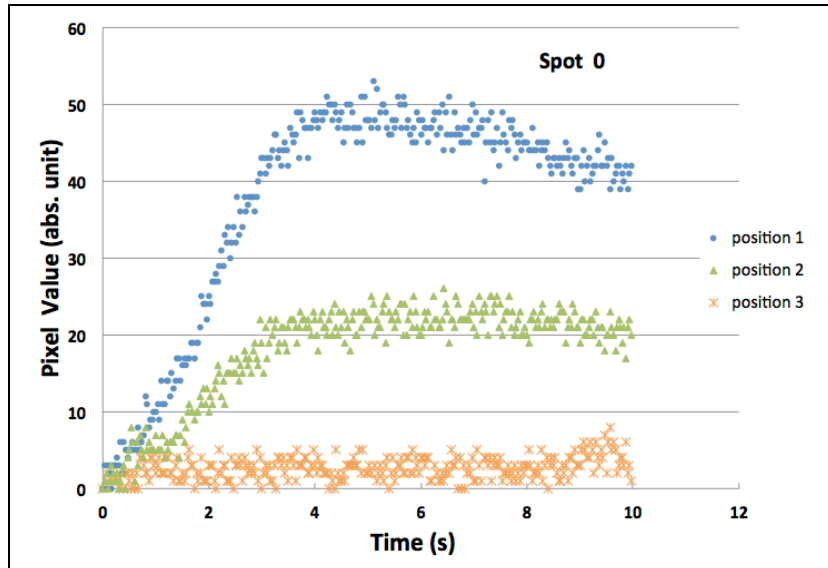

Fig. 4a. Contrast IR signals on spot 0 for the cases in which the ultrasound transducer was placed at positions 1,2 , and 3 .

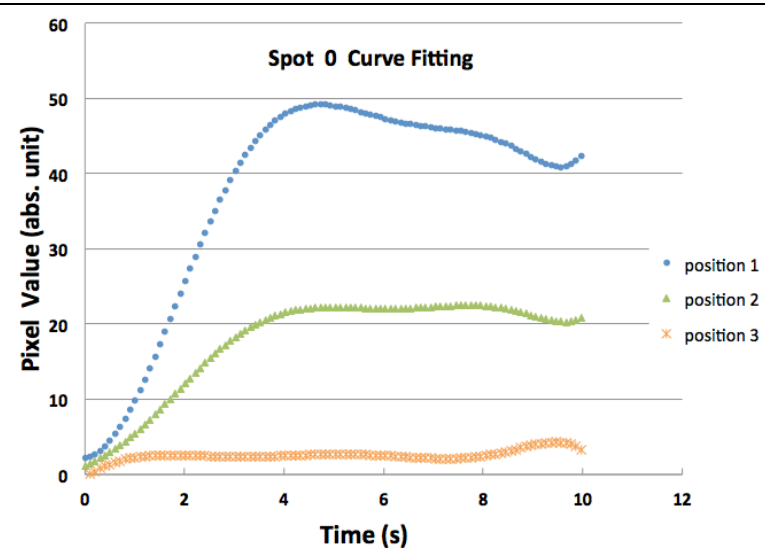

Fig. 4 b. Curve fitting of the data in Figure $2 a$ on spot 0 for the cases in which the ultrasound transducer was placed at positions 1,2 , and 3 .

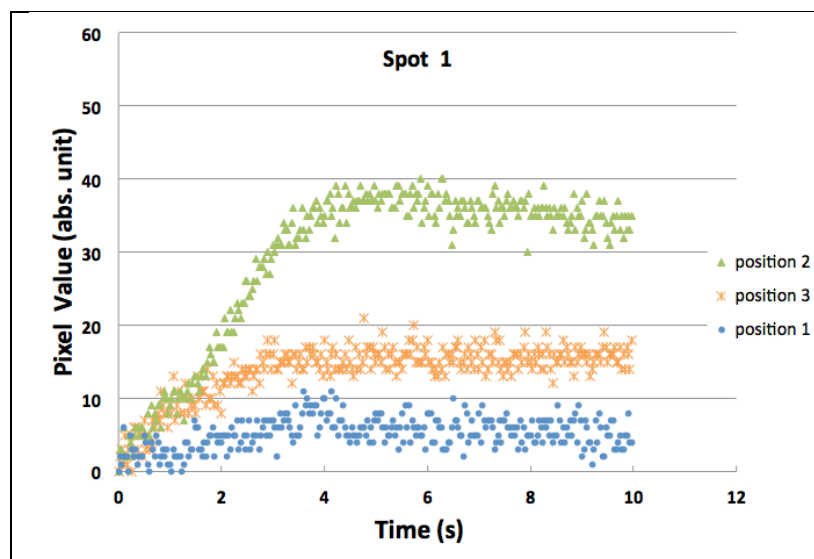

Fig. 5a. Contrast IR signals on spot 0 for the cases in which the ultrasound transducer was placed at positions 1,2 , and 3 .

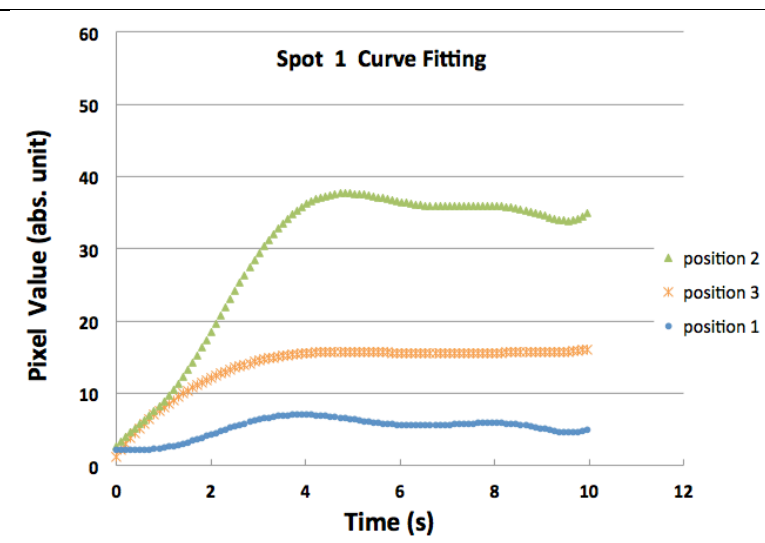

Fig. 5b. Curve fitting of the data in Figure $2 a$ on spot 0 for the cases in which the ultrasound transducer was placed at positions 1, 2, and 3.

\section{Conclusions}

This study is to aim at the effect of the structures and the relative positions of the sound source and the defects. The effect of those factors are clearly demonstrated in this study. The IR signals of defects can change largely. It has been shown that the acoustic behavior of composites is quite different from that of metals, and defect detectability could vary dramatically depending on the composite structures and the relative locations between the ultrasound source and the defects. Due to the attenuation of the sound propagation in composites, the ultrasound pulse is typical set longer than that for metal structures, usually close to one second for composites comparing with a small fraction of a second for metals/metal alloy in our experiments. In spite of the attenuation of ultrasound energy propagation, it is shown that Sonic IR Imaging still has the capability of fast wide-area inspection in composite materials and structures, and it can see through disbonds through the regular thickness of aircraft composite skins. 


\section{ACKNOWLEDGMENTS}

This work was sponsored by the Federal Aviation Administration William J. Hughes Technical Center under Contract Number DTFACT-08-C-00043 with Agency Contract Number 437-65061, and in part by Wayne State University.

\section{REFERENCES}

[1] L.D. Favro, Xiaoyan Han, Zhong Ouyang, Gang Sun, Hua Sui, and R.L. Thomas, "Infrared imaging of defects heated by a sonic pulse", Review of Scientific Instruments, Vol. 71, P2418-2421, June 2000.

[2] L.D. Favro, R.L. Thomas, X. Han, Z. Ouyang, G. Newaz, and D. Gentile, "Sonic Infrared Imaging of Fatigue Cracks", Int. Journal of Fatigue 23, S471 (2001).

[3] L.D. Favro, R.L. Thomas, Xiaoyan Han, Zhong Ouyang, Gang Sun, and Hua Sui, "Method for infrared imaging of ultrasonically excited subsurface defects in materials", US patent No. 6,236,049, 2001

[4] L.D. Favro, Xiaoyan Han, Zhong Ouyang, Gang Sun, and R.L. Thomas, "Sonic IR Imaging of Cracks and Delamination", Analytical Sciences, 17, pp451-453, 2001.

[5] X. Han, L.D. Favro, and R.L. Thomas, "Recent Developments In Sonic IR Imaging", Review of Progress in Quantitative Nondestructive Evaluation 22, ed. By D.O. Thompson and D.E. Chimenti, Am. Inst. Phys. CP657, pp. 500-504 (2003).

[6] Xiaoyan Han, Md. Sarwar Islam, G. Newaz, L.D. Favro, and R.L. Thomas, "Simulation of Sonic IR Imaging of Cracks in Metals with Finite-Element Models", AIP, Review of Progress in Quantitative Nondestructive Evaluation, Vol. 25, pp544-549, 2006.

[7] Qi He, Xiaoyan Han, "Crack Detection Using Sonic Infrared Imaging in Steel Structures: Experiments and Theory of Heating Patterns", SPIE, Smart Structures and Materials, Vol. 7292, 72920Q-1-7, 2009

[8] Xiaoyan Han, Md. Sarwar Islam, "Progress on Developing Acoustic-Infrared NDE Imaging: Studying Motions in Crack Faces", SPIE, Smart Structures and Materials 2010, Vol. 7647, pp76473L-1-6.

[9] Michael W. Burke ${ }^{1}$ and Wayne O. Miller" "Vibrothermographic Inspection of Tube Braze Joints and Comparison with Dye Penetrant Inspection", AIP, Review of Progress in Quantitative Nondestructive Evaluation, 2004

[10] Max Rothenfusser " Acoustic Thermography: Vibrational Modes of Cracks and the Mechanism of Heat Generation", Review of Progress in Quantitative Nondestructive Evaluation, 2005

[11] Marco Morbidini, Peter Cawley, Philippe Duffour, Tim Barden, and Darryl Almond, "A new approach for the prediction of the Thermosonic signal from vibration records", Review of Progress in Quantitative Nondestructive Evaluation, 2006

[12] Daniel L. Simon "Vibro-Thermography Crack Tip Temperature Measurements", Review of Progress in Quantitative Nondestructive Evaluation, 2005

[13] Marco Morbidini, P. Cawley, P. Duffour, T. Barden, and D. Almond, "The Relationship Between Crack Damping, Vibration Amplitude and Temperature Rise in Thermosonics NT", QNDE, 2007

[14] Joseph DiMambro, M. Ashbaugh, C. L. Nelson, and F. W. Spencer, "Sonic Infrared (IR) Imaging and Fluorescent Penetrant Inspection Probability of Detection (POD) Comparison", QNDE, Vol. 26, 2007.

[15] Philipp Menner, Christian Spiessberger, Andreas Gleiter, and Gerd Busse, "Aerospace Applications of LockinThermography with Optical, Ultrasonic, and Inductive Excitation" Review of Progress in Quantitative Nondestructive Evaluation, Vol. 30, 2011

[16] Susan E. Burrows and Steve Dixon, Teng Li and Darryl P. Almond, "Combined Ultrasound and Thermography for a Defect Detection System" Review of Progress in Quantitative Nondestructive Evaluation, Vol. 28, 2009

[17] John C. Chen, W. Riddell, K. Lick, and C-H. Wong, "Fracture Mechanics of Crack Growth During Sonic-IR Inspection", Review of Progress in Quantitative Nondestructive Evaluation, 2007

[18] Jeremy B. Renshaw, Stephen D. Holland, R. Bruce Thompson, and David J. Eisenmann,"Synthetic Defects for Vibrothermography", AIP, Review of Progress in Quantitative Nondestructive Evaluation, Vol. 29, 2010.

[19] Zhi Zeng ${ }^{1,2}$, Ning Tao ${ }^{2}$, Lichun Feng ${ }^{2}$, Cunling Zhang ${ }^{2^{*}}$, Xiaoyan $\mathrm{Han}^{3}$, "Support vector machines based defect recognition in SoniclR using 2D heat diffusion features", NDT\&E international, 2011

[20] L.D. Favro, Xiaoyan Han, Zhong Ouyang, Gang Sun, and R.L. Thomas, "Sonic IR Imaging of cracks and Delaminations", the 11th International Conference in Photoacoustic and Photothermal Phenomena, 2000

[21] Ahsan Mian, Xiaoyan Han, Mahmad Islam, Golam Newaz, "Fatigue damage detection in Graphite/Epoxy composites using sonic infrared imaging technique", Journal of Composites Science and Technology, Vol 64, pp657-666, 2004

[22] Xiaoyan Han, "Sonic Infrared Imaging: A novel NDE Technology for Detection of Crack/Delamination/Disbond in Materials and Structures", book chapter in Ultrasonic and Advanced Methods in NDT, edited by C.H. Chen, World Scientific Publishing. 2007.

[23] Xiaoyan Han, L D Favro, and R L Thomas, Invited paper "Sonic IR Imaging of delaminations and disbonds in composites", Journal of Physics D: Applied Physics, 2010

[24] R.L. Thomas, Xiaoyan Han, L. D. Favro, Golam Newaz, invited paper "Infrared Imaging of Defects in Materials with Chaotic Sonic Excitation", the 2010 IEEE International Ultrasonics Symposium, 2010 
[25] X. Han, X. Zhao, D. Zhang, Q. He, Y. Song, A. Lubowicki, G. Newaz, L. D. Favro, and R. L. Thomas, "Studying Impact Damage on Carbon-fiber Reinforced Aircraft Composite Panels with Sonic-IR," AIP, in Review of Progress in Quantitative Nondestructive Evaluation, Vol. 30A, 2010. 